\title{
Correlating EPR and X-ray structural analysis of arsenite-inhibited forms of aldehyde oxidoreductase
}

\author{
Anders Thapper $\cdot$ D. R. Boer $\cdot$ Carlos D. Brondino $\cdot$ \\ José J. G. Moura $\cdot$ Maria J. Romão
}

Received: 10 July 2006/ Accepted: 27 October 2006/ Published online: 1 December 2006

(C) SBIC 2006

\begin{abstract}
Two arsenite-inhibited forms of each of the aldehyde oxidoreductases from Desulfovibrio gigas and Desulfovibrio desulfuricans have been studied by $\mathrm{X}$-ray crystallography and electron paramagnetic resonance (EPR) spectroscopy. The molybdenum site of these enzymes shows a distorted square-pyramidal geometry in which two ligands, a hydroxyl/water molecule (the catalytic labile site) and a sulfido ligand,
\end{abstract}

Electronic supplementary material Supplementary material is available in the online version of this article at http://dx.doi.org/ $10.1007 / \mathrm{s} 00775-006-0191-9$ and is accessible for authorized users.

\footnotetext{
A. Thapper · D. R. Boer · J. J. G. Moura .

M. J. Romão $(\square)$

REQUIMTE-CQFB,

Departamento de Química,

Faculdade de Ciências e Tecnologia,

Universidade Nova de Lisboa,

2829-516 Caparica, Portugal

e-mail: mromao@dq.fct.unl.pt

C. D. Brondino $(\bowtie)$

Departamento de Física,

Facultad de Bioquímica y Ciencias Biológicas,

Universidad Nacional del Litoral,

3000 Santa Fe, Argentina

e-mail: brondino@fbcb.unl.edu.ar
}

\section{Present Address:}

A. Thapper

Molecular Biomimetics,

Department of Photochemistry and Molecular Science,

Uppsala University, Box 523,

75120 Uppsala, Sweden

Present Address:

D. R. Boer

Institute of Molecular Biology,

C/Josep Samitier 1-5,

08028 Barcelona, Spain have been shown to be essential for catalysis. Arsenite addition to active as-prepared enzyme or to a reduced desulfo form yields two different species called A and $\mathrm{B}$, respectively, which show different $\mathrm{Mo}(\mathrm{V})$ EPR signals. Both EPR signals show strong hyperfine and quadrupolar couplings with an arsenic nucleus, which suggests that arsenic interacts with molybdenum through an equatorial ligand. X-ray data of single crystals prepared from EPR-active samples show in both inhibited forms that the arsenic atom interacts with the molybdenum ion through an oxygen atom at the catalytic labile site and that the sulfido ligand is no longer present. EPR and X-ray data indicate that the main difference between both species is an equatorial ligand to molybdenum which was determined to be an oxo ligand in species A and a hydroxyl/water ligand in species $\mathrm{B}$. The conclusion that the sulfido ligand is not essential to determine the EPR properties in both MoAs complexes is achieved through EPR measurements on a substantial number of randomly oriented chemically reduced crystals immediately followed by X-ray studies on one of those crystals. EPR saturation studies show that the electron transfer pathway, which is essential for catalysis, is not modified upon inhibition.

Keywords Molybdenum-containing enzymes · Aldehyde oxidoreductase Xanthine oxidase family Electron paramagnetic resonance $\cdot \mathrm{X}$-ray
Abbreviations
AOR Aldehyde oxidoreductase
DdAOR Aldehyde oxidoreductase from Desulfovibrio desulfuricans ATCC 27774
$D g A O R$ Aldehyde oxidoreductase from Desulfovibrio gigas 


$\begin{array}{ll}\text { EPR } & \text { Electronic paramagnetic resonance } \\ \text { EXAFS } & \text { Extended X-ray absorption fine structure } \\ \text { HEPES } & \begin{array}{l}N \text {-(2-Hydroxyethyl)piperazine- } N^{\prime} \text { - } \\ \text { ethanesulfonic acid }\end{array} \\ \text { PDB } & \begin{array}{l}\text { Protein Data Bank } \\ \text { PEG4K }\end{array} \\ \text { Poly(ethylene glycol) } 4000 \\ \text { QOR } & \text { Quinoline 2-oxidoreductase } \\ \text { Tris } & \text { Tris(hydroxymethyl)aminomethane } \\ \text { XO } & \text { Xanthine oxidase }\end{array}$

\section{Introduction}

Aldehyde oxidoreductases (AORs) from sulfatereducing bacteria [aldehyde:acceptor oxidoreductase (flavin adenine dinucleotide independent) or aldehyde dehydrogenase or MOP, EC 1.2.99.7] are members of the xanthine oxidase (XO) family of mononuclear molybdenum enzymes [1-5]. The enzymes of this family generally catalyze hydroxylation reactions where, unlike other hydroxylases, water rather than dioxygen is the source of the oxygen atom inserted into the substrate $[1,6]$.

The AOR isolated from the sulfate reducer Desulfovibrio gigas (DgAOR) was the first mononuclear molybdenum enzyme to be structurally characterized at near-atomic resolution $(1.28 \AA)$ [7, 8]. Several crystal structures of other members of the family have also been determined [9-14]. The combination of structural and spectroscopic data on enzymes of this family has shown that the active site consists, in the $\mathrm{Mo}(\mathrm{VI})$ state, of a molybdenum atom in a squarepyramidal geometry that coordinates the two sulfur atoms from one pyranopterin ligand, one oxo ligand, one hydroxyl/water molecule and one sulfido ligand. It has been found that in a number of crystal structures the sulfido ligand is replaced by a second oxo ligand, and these structures represent the so-called desulfo form of the enzyme (Fig. 1a). In contrast to XO, there is no structural evidence supporting a sulfido ligand in as-prepared active AORs from sulfate-reducing bacteria. However, its presence was suggested from spectroscopic studies (mainly electron paramagnetic resonance, EPR), which showed that most of the EPR properties of active $D g$ AOR or AOR from Desulfovibrio desulfuricans ATCC 27774 (DdAOR) are similar to those of active XO $[15,16]$. In addition, cyanide acts as an inhibitor of $D g$ AOR activity [17]; this is also the case in XO (where the cyanide is proposed to react with the sulfido ligand), and the desulfo form of $D g$ AOR regains activity after resulfuration
[18]. The position of the sulfido ligand in the active site has been debated; a crystal structure of "resulfurated" $D g$ AOR crystals showed that the sulfido ligand introduced by adding an excess of sulfide ions was coordinated in the apical position to the molybdenum atom [19] (Fig. 1b). However, the recent crystal structures of quinoline 2-oxidoreductase (QOR) [14] (Fig. 1c) and $\mathrm{XO}$ with a substrate analog bound to the molybdenum atom [13] (Fig. 1d) show this sulfido ligand in the equatorial plane. The interaction of the enzymes with substrate has been shown to occur with the hydroxyl/ water ligand in the equatorial position (cf. Fig. 1a), and the sulfido ligand is proposed to be involved in the enzymatic reaction by abstracting the hydrogen atom from the carbon atom where hydroxylation takes place $[20,21]$.

In addition to the molybdenum active site, enzymes of the XO family of enzymes contain two iron-sulfur clusters of the [2Fe-2S] type named FeS I and FeS II, which are assumed to be electron transfer centers. FeS I, the proximal center, is closer to the Mo site and is buried inside the protein, whereas FeS II, the distal center, is situated near the surface of the protein. Both iron-sulfur centers and a fraction of the molybdenum ions are paramagnetic in the reduced state of the protein and, in addition, show intercenter magnetic couplings [25-27]. The currently accepted mechanism implies substrate interaction with the Mo center, which is then reduced from $\mathrm{Mo}(\mathrm{VI})$ to $\mathrm{Mo}(\mathrm{IV})$, followed by a two electron transfer to an external electron acceptor, in a process mediated by the two iron-sulfur centers. Other members of the XO family, like XO, QOR and $\mathrm{CO}$ dehydrogenase, contain an additional domain with a flavin adenine dinucleotide group bound, which extends the electron transfer pathway from the active site to the external electron acceptor $[1,4]$.

Small molecules such as arsenite, alcohols, aldehydes and glycols can react directly with the molybdenum site and constitute important structural probes for screening the coordination of the active site. Particularly, the inhibition of these enzymes with arsenite has been extensively studied by different spectroscopic techniques [22-24, 28]. After incubation with arsenite followed by dithionite reduction, these enzymes develop a typical EPR spectrum, which was interpreted assuming both quadrupolar and hyperfine interaction of a single arsenic nucleus $(I=3 / 2)$ with the $\mathrm{Mo}(\mathrm{V})$ ion $(S=1 / 2)$ [22]. Different models have been proposed for the arsenite binding to the Mo site. George and Bray [22] postulated a structure based on EPR data, where the sulfido ligand bridges the As and the Mo atoms (Fig. 1e). The sulfur bridge was also suggested by Cramer and Hille [28] for the interaction between 
<smiles>O=[W]1(O)Sc2ssc2S1</smiles><smiles></smiles><smiles>O=[W]1Sc2ssc2S1(=O)=S</smiles><smiles>O=[W]1(O)Sc2ssc2S1</smiles>

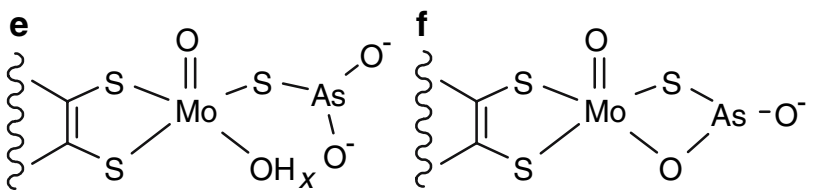<smiles>O=[As]([O-])O[W]1(=O)Sc2ssc2S1</smiles>
$x=1,2$<smiles>Nc1nc2c(c(=O)[nH]1)NC1C([S-])=C([S-])C(COP(=O)([O-])[O-])OC1N2</smiles>

pyranopterin

Fig. 1 Geometries found in active sites of xanthine oxidase $(X O)$ family members. a Aldehyde oxidoreductase $(A O R)$ from Desulfovibrio gigas $(D g A O R)$ as found in the high-resolution crystal structure by Rebelo et al. [8, 9]. b "Resulfurated" $D g A O R$, as determined by Huber et al. [19]. c Quinoline 2oxidoreductase, by Bonin et al. [14]. d XO with substrate analog bound [ $\mathrm{R}$ is 4-(5-pyridin-4-yl-1H-[1,2,4]triazol-3-yl)pyridine-2carbonitrile], by Okamoto et al. [13]. e Suggested structure of arsenite-inhibited $\mathrm{XO}$ based on the electron paramagnetic resonance $(E P R)$ study by George and Bray [22]. f Suggested structure of arsenite-inhibited XO based on the study by Hille et al. [23]. $\mathbf{g}$ Structure of arsenite-inhibited AOR as determined by X-ray crystallography by Boer et al. [24]. h Pyranopterin

As and Mo from extended X-ray absorption fine structure (EXAFS) data of reduced samples of XO reacted with arsenite. A similar structure, but with a double bridge, was proposed earlier by Hille et al. [23] (Fig. 1f). In contrast, we showed in a recent X-ray diffraction study of $D g$ AOR crystals soaked with an arsenite solution [24] that the molybdenum atom in the desulfo form of $D g A O R$ interacts with arsenite through an equatorial oxygen ligand situated in the proposed substrate binding site, suggesting that the sulfido ligand is not directly involved in the coordina- tion of the arsenic atom in the case of arsenite inhibition of $D g$ AOR (Fig. $1 \mathrm{~g}$ ).

We report here X-ray and EPR studies performed on two different arsenite-inhibited forms of AOR. The studies were carried out using $D g$ AOR and $D d$ AOR, in which the initial redox state of the enzyme determines which inhibited species is formed. The two forms were used to determine how molybdenum and arsenite interact, and to clarify if the sulfido ligand takes part in this interaction. This was accomplished by measuring the EPR properties of arsenite-inhibited AOR in frozen polycrystalline samples, which were then used for $\mathrm{X}$-ray experiments. The concomitant use of these two techniques allows us to link unambiguously the EPR properties of the inhibited forms of the enzyme with their molecular structure. We also investigated the integrity of the electron transfer pathway upon inhibition in the two inhibited forms of the enzyme. We discuss these results in view of the proposed general mechanism of the XO family of enzymes.

\section{Materials and methods}

Protein purification

AOR was purified from Desulfovibrio gigas as described by Moura et al. [29, 30] and from Desulfovibrio desulfuricans ATCC 27774 as described by Duarte et al. [16].

\section{Preparation of samples for EPR spectroscopy}

For each aldehyde reductase, two different EPR signals of arsenite-inhibited AOR were obtained, which we will refer to as signal A and signal $\mathrm{B}$. To produce signal A, a solution of sodium arsenite (final concentration $5 \mathrm{mM}$ ) was added to a $200 \mu \mathrm{M}$ sample of AOR in $10 \mathrm{mM}$ tris(hydroxymethyl)aminomethane (Tris)$\mathrm{HCl}$ buffer ( $\mathrm{pH}$ 7.6) and the mixture was incubated for 15 min. A 50-fold excess of sodium dithionite was added to the sample under an argon atmosphere and after $5 \mathrm{~min}$ of reduction a signal from $\mathrm{Mo}(\mathrm{V})$ interacting with arsenite was detected with EPR. To produce signal $\mathrm{B}$, a $200 \mu \mathrm{M}$ sample of AOR in $10 \mathrm{mM}$ Tris-HCl buffer ( $\mathrm{pH}$ 7.6) was reduced with a 50-fold excess of sodium dithionite under an argon atmosphere until only the "slow"-type EPR signal was observed at $140 \mathrm{~K}$ [30]. Sodium arsenite was then added, to a final concentration of $5 \mathrm{mM}$. After $1 \mathrm{~h}$ of incubation, a new EPR signal was detected. Also, using the same methods, samples that exhibited EPR signals A and B were prepared in Tris- $\mathrm{DCl}$ buffered $\mathrm{D}_{2} \mathrm{O}$ solutions, as 
a control with respect to the assignment of exchangeable protons.

\section{EPR spectroscopy}

EPR spectra were recorded with a Bruker EMX spectrometer equipped with an Oxford Instruments continuous-flow cryostat. The experimental conditions were as follows: microwave frequency, $9.65 \mathrm{GHz}$; modulation field, $100 \mathrm{KHz}$; modulation amplitude, $4 \mathrm{G}$; microwave power, $2 \mathrm{~mW}$ at $140 \mathrm{~K}$ and $0.2 \mathrm{~mW}$ at $20 \mathrm{~K}$. As-isolated $D d \mathrm{AOR}$ and $D g$ AOR present a $\mathrm{Mo}(\mathrm{V})$ EPR signal normally referred to as the "resting" signal (not shown). This signal is not affected by addition of $\mathrm{AsO}_{2}^{-}$nor by reduction with dithionite when producing signal A. In contrast, the resting signal disappears when the "slow" signal is produced by long reduction using sodium dithionite. The contribution of the resting signal was subtracted in all the spectra showing signal A (Figs. 2, 4, spectra a, c). Computer simulations of the EPR spectra were performed using the program QPOWA [31, 32].

\section{Preparation of samples for crystallography}

Single crystals of arsenite-inhibited AOR were prepared using three methods: (1) by soaking single crystals of AOR in arsenite-containing solutions [24], (2) by growing crystals using a protein sample from an EPR tube giving signal A (DgAOR-A) and (3) by growing crystals from an EPR tube giving signal $\mathrm{B}$ (DgAOR-B). For all methods, the crystals were grown using the sitting-drop method, during which no special care was taken to prevent the samples from coming into contact with air. The structures of the crystals obtained with method 1 will be identified according to the EPR signal detected (see later) followed by asterisk. A flow chart summarizing the sample preparation for the various experiments described in this article is given in Scheme 1.

Preparation of $D g$ AOR crystals with method 1 and manipulation for EPR and X-ray experiments

A purified protein sample of $D g$ AOR was concentrated to $12 \mathrm{mg} / \mathrm{mL}$ in $10 \mathrm{mM}$ Tris- $\mathrm{HCl} \mathrm{pH} 7.6$ buffer. Crystals were obtained over a period of 3 weeks using vapor diffusion on sitting drops using a 5:3 (v/v) mixture of the protein solution and a crystallization solution of $0.2 \mathrm{M}$ $\mathrm{MgCl}_{2}, 0.1 \mathrm{M} \mathrm{N}$-(2-hydroxyethyl)piperazine- $N$ '-ethanesulfonic acid (HEPES) pH 7.6 and 30\% v/v 2-propanol [7, 33]. After the crystals had reached their maximum dimensions $(0.3 \mathrm{~mm} \times 0.15 \mathrm{~mm} \times 0.15 \mathrm{~mm})$, a cryo-protectant harvesting buffer was added, which contained the solutes of the crystallization solution at the concentrations mentioned above, as well as 30\% (w/ v) poly(ethylene glycol) 4000 (PEG4K). After leaving the crystals to equilibrate in the harvesting buffer for 4 days, crystals from 45 drops were collected in a small vial and the solution in the vial was replaced by a soaking solution containing $0.2 \mathrm{M} \mathrm{MgCl}_{2}, 0.1 \mathrm{M} \mathrm{HEPES}$ $\mathrm{pH} 7.6,40 \%$ PEG4K and $50 \mathrm{mM} \mathrm{NaAsO}_{2}$. Special care was taken not to expose the crystals to osmotic shock in the process. A total of about 400 crystals were collected for analysis.

The crystals were then transferred into an EPR tube and kept under an argon atmosphere on ice for $30 \mathrm{~min}$, after which the tube was frozen for an EPR experiment.
Fig. 2 The two arseniteinhibited EPR signals produced with AOR. $a$ Signal A obtained in arseniteinhibited AOR from Desulfovibrio desulfuricans ATCC $27774(D d A O R)$ in buffered $\mathrm{H}_{2} \mathrm{O}$ and $\mathrm{D}_{2} \mathrm{O}$ solutions at $140 \mathrm{~K}$ together with the simulation ( $\mathrm{sim})$. The EPR spectra obtained in $D g$ AOR are very similar to those of DdAOR. $b$ Signal B of arsenite-inhibited $D g A O R$, in buffered $\mathrm{H}_{2} \mathrm{O}$ and $\mathrm{D}_{2} \mathrm{O}$ solutions together with simulations. Similar signals A and $\mathrm{B}$ were obtained in both $D g$ AOR and $D d$ AOR (see figures in the supplementary material)

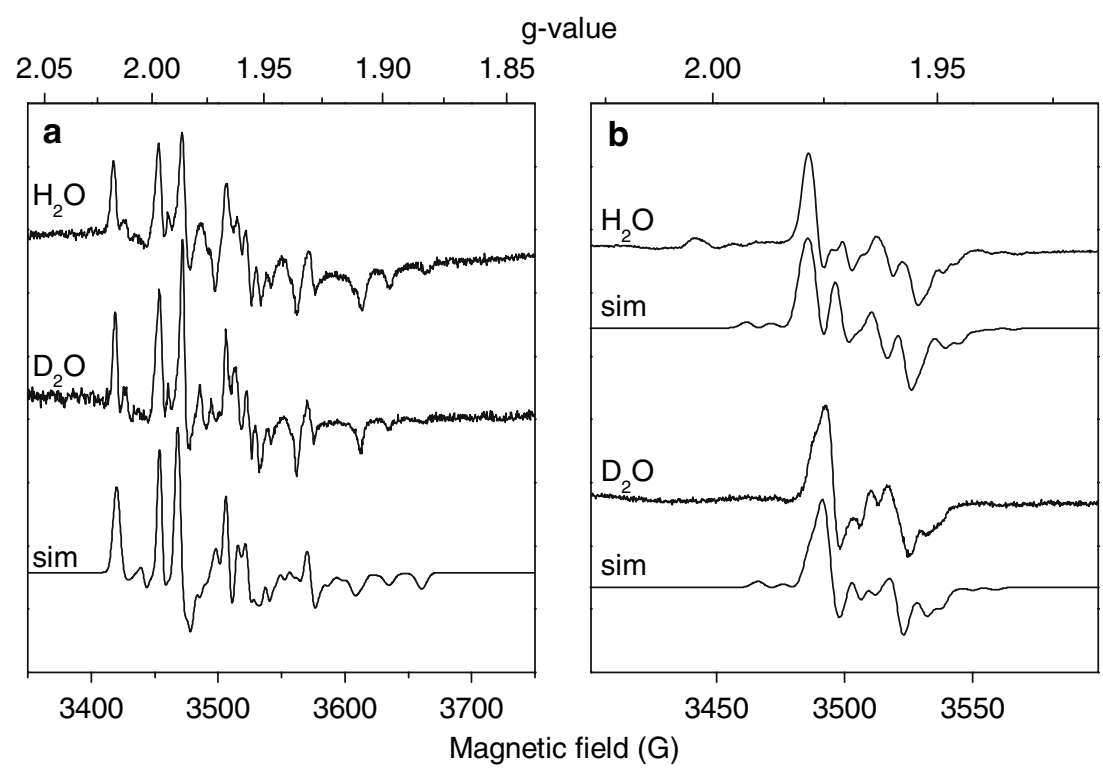


Scheme 1 Flow chart showing how samples were prepared for the various experiments described in this article. Method 1 describes the preparation of crystals for powder electron

paramagnetic resonance $(E P R)$ and X-ray diffraction experiments used in the correlation of the EPR signal and atomic structure.

Methods 2 and 3 involve the use of, respectively, buffered aqueous samples containing species $\mathrm{A}$ and $\mathrm{B}$ for subsequent crystallization experiments

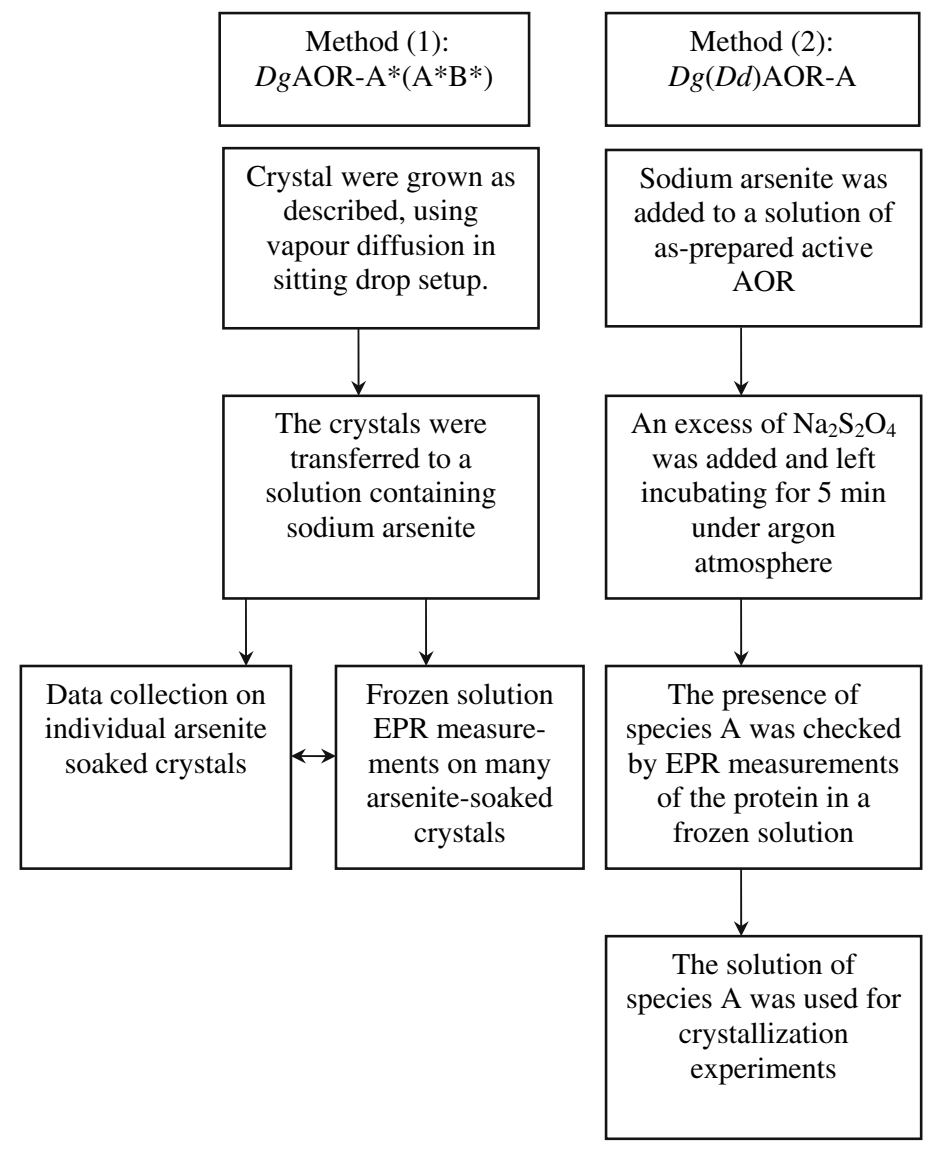

The sample was thawed and kept on ice and a large excess of sodium dithionite was added under argon and it was left to reduce for $10 \mathrm{~min}$, followed by freezing and EPR spectra acquisition.

The tube was once again thawed and a small number of crystals were removed from the tube and immediately frozen in a stream of liquid nitrogen at $100 \mathrm{~K}$ (DgAOR-A*). In parallel, the EPR tube was immediately put back under argon and the contents were left to reduce for another $4 \mathrm{~h}$ on ice, after which the sample was frozen for EPR measurements. Finally the sample was thawed and the withdrawal procedure was repeated $(D g$ AOR-A*B*). EPR measurements at low temperatures $(20 \mathrm{~K})$ of the reduced samples before and after withdrawing the crystals for X-ray analysis showed the FeS EPR signals with the same intensity, indicating that the sample remained under reducing conditions even when withdrawing the crystals. EPR experiments on the sample before dithionite addition showed the resting signal like frozen solutions of aspurified samples (not shown). This signal was subtracted in all the spectra.

A small number of the crystals removed in each thawing were mounted and tested for diffraction on an in-house ENRAF-NONIUS rotating-anode diffractometer using $\mathrm{Cu} \mathrm{K} \alpha$ radiation. For one crystal withdrawn after $10 \mathrm{~min}\left(\mathrm{DgAOR}-\mathrm{A}^{*}\right)$, a dataset to $2.35 \AA$ was collected at $100 \mathrm{~K}$, using a MAR fluorescence detector. The data were indexed, recorded, integrated and processed using mosflm6.2.3. [34]. Structure factors were generated using the program TRUNCATE and a subset of $5 \%$ of the total number of unique reflections was used for cross-validation purposes obtained with the script UNIQUEIFY, both from the CCP4 package, version 4.2.2 [35]. The structure was refined with REFMAC5.2.0005 [36] using the Protein Data Bank (PDB) entry 1VLB as the initial model, starting with a rigid-body refinement followed by restrained refinement. The final $R_{\text {cryst }}$ and $R_{\text {free }}$ were 17.8 and $22.0 \%$, respectively (Table 1 ). One of the crystals retrieved after $4 \mathrm{~h}$ (DgAOR$\left.\mathrm{A}^{*} \mathrm{~B}^{*}\right)$ was subjected to a similar procedure. Relevant data collection and refinement data are summarized in Table 1.

\section{Preparation of $D g$ AOR-A and $D g$ AOR-B crystals}

Samples of $D g$ AOR that produced pure EPR signals A and $\mathrm{B}$, respectively, were used to grow crystals by removing some of the sample solution from the EPR 
Table 1 Crystal and refinement parameters of the arsenite-inhibited structures of aldehyde oxidoreductase (AOR) from Desulfovibrio gigas $(D g A O R)$ and AOR from Desulfovibrio desulfuricans ATCC 27774 (DdAOR)

\begin{tabular}{|c|c|c|c|c|c|}
\hline Data collection details & $D g$ AOR-B $^{\mathrm{a}}$ & $D g$ AOR-A $*^{\mathrm{b}}$ & $D g$ AOR $-\mathrm{A}^{*} \mathrm{~B} * \mathrm{~b}$ & $D d \mathrm{AOR}_{-} \mathrm{A}^{\mathrm{c}}$ & $D d \mathrm{AOR}-\mathrm{B}^{\mathrm{a}}$ \\
\hline Unit cell $(\AA)$ & $\begin{array}{c}a=b=142.89 \\
\quad c=161.64\end{array}$ & $\begin{array}{c}a=b=143.04 \\
\quad c=161.58\end{array}$ & $\begin{array}{c}a=b=143.36 \\
c=162.10\end{array}$ & $\begin{array}{c}a=b=159.65 \\
c=166.52\end{array}$ & $\begin{array}{c}a=b=159.76 \\
c=166.36\end{array}$ \\
\hline Space group & $P 6_{1} 22$ & $P 6_{1} 22$ & $P 6_{1} 22$ & $P 6_{1} 22$ & $P 6_{1} 22$ \\
\hline Wavelength $(\AA)$ & 0.931 (ESRF) & 1.542 (in-house) & 1.542 (in-house) & $0.931(\mathrm{ESRF})$ & $0.931(\mathrm{ESRF})$ \\
\hline Resolution range & $\begin{array}{l}20-1.45(1.50- \\
1.45)\end{array}$ & $\begin{array}{l}25-2.35(2.48- \\
2.35)\end{array}$ & $\begin{array}{l}25-3.05(3.21- \\
3.05)\end{array}$ & $20-2.2(2.32-2.2)$ & $20-2.0(2.11-2.0)$ \\
\hline No. of observed reflections & $325,446(30,794)$ & $171,814(19,363)$ & $64,127(9,298)$ & $684,714(93,663)$ & $894,500(118,690)$ \\
\hline No. of unique reflections & $169,887(15,967)$ & $28,895(4,125)$ & $18,206(2,575)$ & $63,725(9,174)$ & $84,104(11,943)$ \\
\hline Completeness (\%) & $99.6(97.8)$ & $97.2(81.4)$ & $95.8(95.0)$ & $99.9(100.0)$ & $99.8(99.7)$ \\
\hline$R_{\text {merge }}(\%)^{\mathrm{d}}$ & $3.5(27.2)$ & $8.6(27.7)$ & $17.7(36.6)$ & $9.5(74.7)$ & $10.8(71.2)$ \\
\hline$I / \sigma(I)$ & $16.61(3.1)$ & $13.3(5.2)$ & $8.6(3.0)$ & $17.2(3.2)$ & $13.8(3.0)$ \\
\hline Redundancy & $3.7(3.1)$ & $4.3(4.0)$ & $3.5(3.6)$ & $10.7(10.2)$ & $10.6(9.8)$ \\
\hline \multicolumn{6}{|l|}{ Model details } \\
\hline Protein atoms & 6,890 & 6,890 & 6,886 & 6,854 & 6,854 \\
\hline$[2 \mathrm{Fe}-2 \mathrm{~S}]$ clusters & 8 & 8 & 8 & 8 & 8 \\
\hline Magnesium atoms & 3 & 3 & 3 & 0 & 0 \\
\hline Chlorine atoms & 3 & 3 & 3 & 0 & 0 \\
\hline MCD atoms & 48 & 48 & 48 & 48 & 48 \\
\hline Arsenite atoms & 3 & 3 & 3 & 3 & 3 \\
\hline Water $\mathrm{O}$ atoms & 1,002 & 875 & 480 & 192 & 192 \\
\hline \multicolumn{6}{|l|}{ Refinement details } \\
\hline $\begin{array}{l}\text { No. of reflections used in } \\
\text { refinement }\end{array}$ & 161,596 & 37,968 & 17,238 & 60,375 & 79,905 \\
\hline$R$ factor for all data (\%) & 15.8 & 17.8 & 18.6 & 22.0 & 22.9 \\
\hline$R_{\text {free }}(\%)$ & 18.0 & 22.0 & 26.3 & 24.4 & 25.3 \\
\hline \multirow{3}{*}{$\begin{array}{l}\text { Root mean square deviation of } \\
\text { target values }\end{array}$} & Bonds 0.010 & Bonds 0.005 & Bonds 0.006 & Bonds 0.012 & Bonds 0.010 \\
\hline & Angle 1.453 & Angle 0.953 & Angle 0.954 & Angle 1.467 & Angle 1.413 \\
\hline & Chiral 0.098 & Chiral 0.068 & Chiral 0.069 & Chiral 0.093 & Chiral 0.093 \\
\hline $\begin{array}{l}\text { Mean isotropic equivalent } B \\
\text { factor }\left(\AA^{2}\right)\end{array}$ & 12.5 & 23.5 & 21.1 & 35.0 & 31.6 \\
\hline Solvent content $(\%)$ & 42.4 & 43.1 & 46.4 & 59.7 & 59.8 \\
\hline
\end{tabular}

See "Preparation of samples for crystallography" for designations of the structures. Values in parentheses are for the highest-resolution shell

${ }^{\text {a }}$ Crystals grown from the sample giving signal B

${ }^{\text {b }}$ Crystals treated with $\mathrm{AsO}_{2}{ }^{-}$followed by $10 \mathrm{~min}\left(D g \mathrm{AOR}-\mathrm{A}^{*}\right)$ and $4 \mathrm{~h}\left(D g \mathrm{AOR}-\mathrm{A}^{*} \mathrm{~B}^{*}\right)$ of dithionite reduction

c Crystals grown from the sample giving signal A

${ }^{\mathrm{d}} R_{\mathrm{sym}}=\Sigma_{h} \Sigma_{i}\left|\left\langle I_{h i}\right\rangle-\left\langle I_{h}\right\rangle\right| \Sigma_{h} \Sigma_{i} I_{h i}$

tubes for use in setting up hanging-drop vapor-diffusion experiments as described earlier. Crystals appeared for both types of experiments, but those from $D g$ AOR that produced EPR signal A were small (dimensions $0.02 \mathrm{~mm} \times 0.01 \mathrm{~mm} \times 0.01 \mathrm{~mm}$ ) and did not diffract. The crystals obtained from $D g$ AOR giving signal B (DgAOR-B) diffracted to near-atomic resolution $(1.45 \AA)$ at the ID14-EH3 beamline of the ESRF synchrotron in Grenoble at $100 \mathrm{~K}$. A full dataset was measured to $1.2 \AA$ at a wavelength of $0.931 \AA$ using a MarCCD 165-mm detector. The data were indexed, recorded, integrated and processed using the XDS package [37] to a resolution of $1.45 \AA$. Structure factors were generated using the program TRUNCATE and a subset of $5 \%$ of the total number of unique reflections was used for cross-validation purposes obtained with the script UNIQUEIFY, both from the CCP4 package, version 4.2.2 [35]. Table 1 summarizes the data collection and refinement details. Using the crystal structure from the PDB with code 1SIJ, we refined the structure with REFMAC5.2.0005 [36] starting with five cycles of rigid-body refinement, followed by restrained refinement. Water molecules were refined in seven cycles of refinement using arpwaters [38] and were subjected to restrained refinement using REFMAC5.2.0005 [36]. The final $R_{\text {cryst }}$ and $R_{\text {free }}$ were 15.8 and $18.0 \%$, respectively (Table 1 ). The calculation of double-difference and difference maps using the arsenite-free atomic-resolution structure 1VLB excluded the occurrence of model bias in the arsenitebinding region. The structure has been deposited in the PDB in Brookhaven with reference code 1ZCS. 
Preparation of DdAOR-A and DdAOR-B crystals

Crystalline arsenite-inhibited forms of $D d$ AOR were prepared from EPR samples exhibiting pure signal A or $\mathrm{B}$, as described for $D g$ AOR-A and $D g$ AOR-B, using conditions similar to those described previously [9]. These crystals are referred to as DdAOR-A and DdAOR-B, respectively. Data were collected at the BM14 beamline of the ESRF synchrotron in Grenoble at $100 \mathrm{~K}$ and a wavelength of $0.931 \AA$. Data to 2.2 and $2.0 \AA$ were indexed, recorded, integrated and processed using mosflm [34] for the crystals derived from samples giving signals A and B, respectively (Table 1). Structure factor generation and subset designation were performed as described earlier. Both structures were refined with REFMAC5.2.0005 [36] using the PDB entry 1DGJ as the initial model, starting with a rigid-body refinement. The arsenite moiety clearly showed up in the both the $2 F_{\mathrm{o}}-F_{\mathrm{c}}$ and $F_{\mathrm{o}}-F_{\mathrm{c}}$ maps and was added accordingly, followed by ten cycles of restrained refinement. The final $R_{\text {cryst }}$ and $R_{\text {free }}$ were 22.0 and $24.4 \%$ for DdAOR-A and 22.9 and $25.3 \%$ for DdAOR-B, respectively (Table 1).

\section{Results}

EPR properties of $\mathrm{Mo}(\mathrm{V})$ species

As stated already, proteins of the $\mathrm{XO}$ family incubated with sodium arsenite and reduced with sodium dithionite yield an EPR-active $\mathrm{Mo}(\mathrm{V})$ species. For bovine milk XO this species has been characterized by X-band and Q-band EPR. The signals were simulated by assuming both hyperfine and quadrupolar coupling of a single arsenic nucleus $(I=3 / 2)$ to a $\mathrm{Mo}(\mathrm{V})$ ion $(S=1 / 2)$ (EPR parameters in Table 2) [22]. A similar signal (signal A) was obtained in DgAOR [24] and DdAOR (Fig. 2, panel a) by reducing the enzyme for about 10-15 min. Figure 2, panel a shows signal A from $D d$ AOR at $9.65 \mathrm{GHz}$ and $140 \mathrm{~K}$ together with a simulation (EPR parameters in Table 2). The strong hyperfine and quadrupolar coupling suggests that the arsenic atom is in close interaction with the $\mathrm{Mo}(\mathrm{V})$ ion ground-state orbital having the unpaired electron, which is usually assumed to be in the plane of the equatorial ligands to the molybdenum atom [39]. Signal A obtained in a buffered $\mathrm{D}_{2} \mathrm{O}$ solution shows no significant differences, indicating that the species giving signal A does not contain exchangeable protons associated with ligands of the first coordination sphere of the $\operatorname{Mo}(\mathrm{V})$ ion, and suggesting that the interaction between the molybdenum and the arsenic atoms involves the position of the hydroxyl/water ligand (cf. Fig. 1a).

In contrast to what was reported for XO [22], desulfo forms of $D g$ AOR and $D d$ AOR, such as those obtained when reduced extensively with dithionite [40], can react with arsenite yielding a new EPR signal (signal B). The lineshape of this signal is completely different with respect to that of signal A. The overall lineshape of signal $\mathrm{B}$ could be simulated assuming non-collinear $\mathbf{A}$ and $\mathbf{P}$

Table 2 Parameters used for simulation of the electron paramagnetic resonance signals at X-band obtained in arsenite-inhibited xanthine oxidase $(X O)$, and $D g A O R$ and $D d A O R$

\begin{tabular}{|c|c|c|c|c|c|c|c|c|}
\hline Enzyme & Signal & Axis & $g$ values $^{\mathrm{a}}$ & $\mathbf{A}(\mathrm{MHz})^{\mathrm{a}}$ & $\mathbf{P}(\mathrm{MHz})^{\mathrm{a}}$ & $\begin{array}{l}\text { Half linewidths } \\
(\mathrm{MHz})\end{array}$ & $\begin{array}{l}\text { A Euler angles } \\
(\alpha, \beta, \gamma) \text { (degrees) }\end{array}$ & $\begin{array}{l}\text { P Euler angles } \\
(\alpha, \beta, \gamma) \text { (degrees) }\end{array}$ \\
\hline \multirow[t]{3}{*}{$\mathrm{XO}^{\mathrm{b}}$} & \multirow[t]{3}{*}{ As-inhibited } & 1 & 1.9258 & -90 & -10 & 12 & \multirow[t]{3}{*}{$(15,0,0)$} & \multirow[t]{3}{*}{$(15,0,0)$} \\
\hline & & 2 & 1.9732 & -40 & 27 & 6 & & \\
\hline & & 3 & 1.9718 & 128 & -17 & 6 & & \\
\hline \multirow[t]{3}{*}{$D g \mathrm{AOR}^{\mathrm{c}}$} & \multirow[t]{3}{*}{ A } & 1 & 1.922 & 120 & -9 & 12 & \multirow[t]{3}{*}{$(0,0,0)$} & \multirow[t]{3}{*}{$(30,0,0)$} \\
\hline & & 2 & 1.979 & 60 & 19 & 6 & & \\
\hline & & 3 & 1.972 & 136 & -10 & 9 & & \\
\hline \multirow[t]{3}{*}{ DdAOR } & \multirow[t]{3}{*}{ A } & 1 & 1.922 & 120 & -8 & 12 & \multirow[t]{3}{*}{$(0,0,0)$} & \multirow[t]{3}{*}{$(30,0,0)$} \\
\hline & & 2 & 1.979 & 60 & 18 & 6 & & \\
\hline & & 3 & 1.972 & 136 & -10 & 9 & & \\
\hline \multirow[t]{3}{*}{$D g$ AOR } & \multirow[t]{3}{*}{ B } & 1 & 1.958 & $22(40)$ & -17 & 7 & & \\
\hline & & 2 & 1.968 & $47(12)$ & 19 & 8 & \multirow{2}{*}{$\begin{array}{l}(135,0,0) \mathrm{As} \\
(0,0,0) \mathrm{H}\end{array}$} & $(2,0,0)$ As \\
\hline & & 3 & 1.970 & $23(37)$ & -2 & 10 & & $(0,0,0) \mathrm{H}$ \\
\hline
\end{tabular}

$\mathbf{A}$ and $\mathbf{P}$ are the hyperfine and quadrupolar tensors, respectively, associated with the arsenic nucleus $(I=3 / 2)$. Values of $\mathbf{A}$ within parentheses for signal $\mathrm{B}$ refer to a solvent-exchangeable proton

${ }^{\text {a }}$ Uncertainties of $g$ values for $D g A O R$ and $D d$ AOR are \pm 0.001 and of $\mathbf{A}$ and $\mathbf{P}$ values are $\pm 10 \%$

b Data from [22]

c Data from [24] 
tensors with respect to the $g$ tensor (Table 2) but with different magnitudes and different relative orientations. In addition, signal B from $D g$ AOR obtained in buffered $\mathrm{D}_{2} \mathrm{O}$ solutions reveals that at least one solvent-exchangeable proton is located near the molybdenum atom (Fig. 2, panel b). As for signal A, the fitting could be only accomplished including a quadrupolar term in the simulation. However, additional measurements at different microwave frequencies are necessary to confirm the uniqueness of the fitting parameters for the two signals. The value of the hyperfine coupling for this proton is within the order of magnitude found in the XO family for equatorial hydroxyl/water ligands bound to the molybdenum [15, 41, 42]. However, two hypotheses can be raised in this case. One of them is that the interaction with the arsenic atom is through the equatorial oxo ligand, leaving the hydroxyl/water ligand intact. The other one is that the interaction takes place at the position of the hydroxyl/ water ligand, as seen in our previously reported structure [24], but with the equatorial oxo ligand being replaced by a hydroxyl/water ligand. Furthermore, signal B also develops in a sample that shows signal A if it is left on ice and under an argon atmosphere, in the presence of excess dithionite for a longer time (more than $30 \mathrm{~min}$, not shown). In this process, signal B appears at approximately 30 min of incubation, reaching its maximum intensity after $1 \mathrm{~h}$, whereas signal A does not change noticeably in intensity. No changes in intensity of either of the signals were observed after further incubation for up to $12 \mathrm{~h}$.

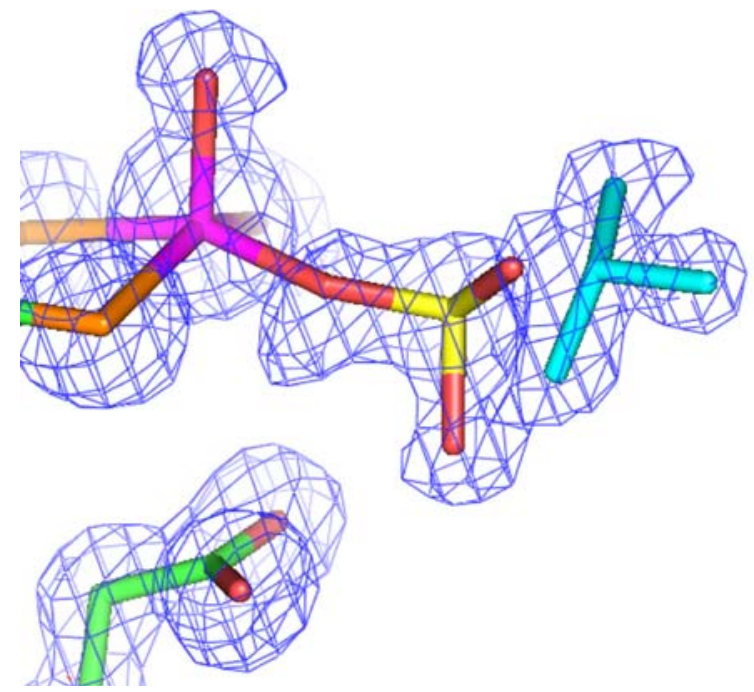

Fig. 3 Stereo picture of the active site and the $2 F_{\mathrm{o}}-F_{\mathrm{c}}$ electron density map of the $1.45-\AA$ resolution structure of an arseniteinhibited sample of $D g A O R$ containing species B, contoured at 1.2 $\sigma$;. Color coding: Mo, pink; As, yellow; O, red; S, orange; C,
Structural data

Crystals of sufficient quality for data collection were obtained for all crystal preparations described in the "Materials and methods" section, except for the crystals grown from a sample taken from an EPR tube containing species A of DgAOR. Notably, crystals grown from a sample taken from an EPR tube containing species B (DgAOR-B) diffracted to nearatomic resolution $(1.45 \AA)$ compared with the $2.3-\AA$ resolution of the previously described structure [24]. The molybdenum atom was present at full occupancy, whereas the arsenic atom was found at $35 \%$ occupancy. The molybdenum sites lacking arsenic atoms showed two 2-propanol molecules, as observed in the desulfo structure of $D g A O R[7,8]$. Figure 3 shows a stereo plot of the electron density in the vicinity of the molybdenum center obtained for DgAOR-B. The general coordination around the molybdenum atom is distorted square pyramidal (all Mo-O distances $1.9 \AA$, Mo-S bonds $2.4 \AA$ ), with the two sulfur atoms from the dithiolene ligand, the bridging oxygen and a second oxygen atom forming a distorted equatorial plane and the apical oxygen atom pointing away from this plane. The molybdenum atom is displaced out of the plane in the direction of the apical oxygen, as described by Huber et al. [19] for the dithiothreitol-reduced form of $D g$ AOR. The arsenic atom is positioned out of this plane in the opposite direction. The Mo-As distance is about $3.3 \AA$, with a Mo-O-As angle of $131^{\circ}$. The $\mathrm{AsO}_{3}$ moiety is pyramidal and is positioned so that one of the

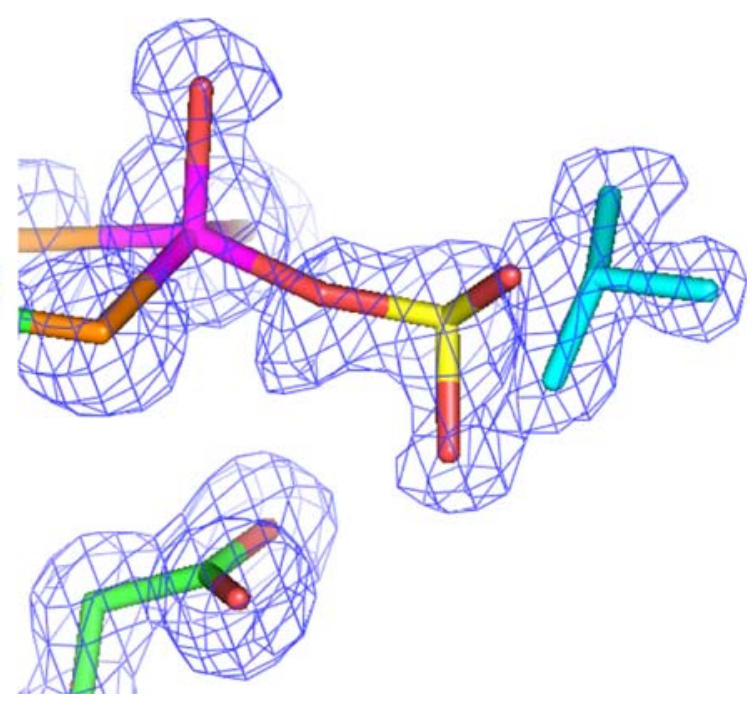

green; N, blue. The partially occupied 2-propanol molecule (see text) is shown in cyan. Glu869 is seen on the lower-left side and is located $2.7 \AA$ from one of the oxygen ligands of $\mathrm{AsO}_{3}$ 
oxygen atoms is within hydrogen-bonding distance $(2.7 \AA)$ of Glu869, a conserved residue essential for catalysis. The lone pair on the arsenic atom is directed out between the two equatorial oxygen atoms on the molybdenum atom. Relevant geometrical parameters are given in Table 3. As discussed earlier, the coordination of the oxygen atoms to the molybdenum atom in this structure is more compatible with hydroxyl ligands than oxo ligands. This is similar to that found by Huber et al. [19] in the structure of reduced forms of $D g$ AOR, suggesting that, though the sample is exposed to air during the crystallization, it is kept in a reduced state.

The As atom was found at $35 \%$ occupancy, similar to what was reported for the previous structure of $D g$ AOR obtained with method 1 [24]. However, the two 2-propanol molecules, which were observed in the desulfo structure obtained from as-purified $D g$ AOR [7, 8], are also present in the arsenite-inhibited structure of $D g$ AOR-B, in contrast to the arseniteinhibited $D g$ AOR obtained with method 1. This occurs because the protein sample was exposed to arsenite before the crystallization experiment and, therefore, the harvesting buffer did not need to be replaced with a 2-propanol-depleted solution. The 2propanol molecule close to the active site in the $D g$ AOR-B structure was found at $65 \%$ occupancy, as its binding site overlaps with that occupied by the arsenite moiety. A striking observation is that the Mo was present at full occupancy in structures from crystals grown using method 3 , whereas it was present at half occupancy in the structure of $D g A O R$ obtained with method 1.

Samples of DdAOR containing arsenite-inhibited species A and B were crystallized according to methods 2 and 3, respectively. The position and the geometry of the Mo-As active site are virtually identical in these two structures (for DdAOR-B, see Fig. S1). In addition, they are essentially the same as those observed in the high-resolution structure of $D g$ AOR-B described above.

As seen above, the structures of DgAOR-A and DdAOR-A show that the sulfido ligand is not coordinated to Mo. However, crystallization of as-prepared DgAOR $[7,8]$ and $D d$ AOR $[9,16]$ samples results in the desulfo form of the enzyme and a similar process could occur in the crystallization of the samples giving signal A. Hence, we cannot confirm with this experiment the involvement of the sulfido ligand in the MoAs complex giving signal A, as previously suggested for milk XO [22, 28]. To address this point, it was considered necessary to approach the problem in a different way, as outlined in the next section.

EPR properties of crystalline arsenite-inhibited AOR and comparison with its molecular structure

In order to correlate the molecular structure of the molybdenum site with the two different EPR signals (signals A and B), we performed EPR experiments on polycrystalline samples (around 400 single crystals randomly oriented) of arsenite-reacted $D g$ AOR obtained with method 1 (see coordination around molybdenum in Fig. 1g) [24], which were then subjected to reducing conditions.

Before analyzing the results, it is important to make some observations on the system we are studying. $D g$ AOR crystallizes in the space group $P 6_{1} 22$ with 12 symmetry-related molybdenum sites per unit cell, which are magnetically nonequivalent for most magnetic field orientations. Hence, in a hypothetical EPR experiment on an oriented single crystal, we should observe 12 symmetry-related spectra at different magnetic field positions owing to the 12 different molybdenum $g$-tensor orientations. Thus, an EPR sample with a large number $(X)$ of single crystals that are randomly oriented contains molybdenum sites with around $12 X$ different spatial orientations. Evidently,

Table 3 Relevant distances (in angstroms) from the molybdenum atom to its ligands in crystal structures of $D g A O R$ and $D d A O R$

\begin{tabular}{|c|c|c|c|c|c|}
\hline & $\begin{array}{l}D g A O R-B \\
(1.45 \AA)^{\mathrm{a}}\end{array}$ & $\begin{array}{l}\text { DgAOR-A* } \\
(2.35 \AA)\end{array}$ & $\begin{array}{l}D g \mathrm{AOR}-\mathrm{A}^{*} \mathrm{~B}^{*} \\
(3.05 \AA)\end{array}$ & $\begin{array}{l}\text { DdAOR-A } \\
(2.2 \AA)\end{array}$ & $\begin{array}{l}D d \text { AOR-B } \\
(2.0 \AA)\end{array}$ \\
\hline $\mathrm{Mo}-\mathrm{O}_{\mu}^{\mathrm{b}}$ & 1.9 & 1.9 & 1.8 & 2.1 & 2.0 \\
\hline $\mathrm{Mo}-\mathrm{O}_{\mathrm{ap}}^{\mathrm{c}}$ & 1.9 & 1.9 & 1.9 & 1.9 & 1.8 \\
\hline $\mathrm{Mo}-\mathrm{O}_{\mathrm{eq}}^{\mathrm{c}^{\mathrm{r}}}$ & 1.9 & 1.9 & 1.9 & 1.9 & 1.9 \\
\hline $\mathrm{Mo}-\mathrm{S} 7^{\mathrm{d}^{\mathrm{t}}}$ & 2.4 & 2.5 & 2.5 & 2.5 & 2.4 \\
\hline Mo-S8 ${ }^{\mathrm{d}}$ & 2.4 & 2.3 & 2.3 & 2.4 & 2.3 \\
\hline
\end{tabular}

\footnotetext{
${ }^{a}$ Higher-resolution limit of the X-ray data

b $\mathrm{O}_{\mu}$ is the bridging oxygen between the Mo and As atoms

${ }^{c} \mathrm{O}_{\mathrm{ap}}$ and $\mathrm{O}_{\mathrm{eq}}$ are the apical and equatorial oxygen atoms, respectively

d S7 and S8 are the sulfur atoms from the pyranopterin
} 
this randomly oriented single-crystal sample gives EPR spectra independent of the magnetic field orientation like the ones obtained in frozen solutions.

Figure 4 shows the EPR spectra obtained on polycrystalline samples at two different incubation times with dithionite, together with signals A (spectrum c) and B (spectrum d) obtained in frozen solutions. Crystals that were grown with method 1 and reduced for 10 min with sodium dithionite yielded only signal A (Fig. 4, spectrum a). The subsequent X-ray diffraction experiment conducted on the crystal withdrawn at this point yielded data to a resolution of $2.35 \AA$ ( DgAOR$A^{*}$ in Table 1) and showed no evidence of a sulfur bound to the molybdenum atom (Fig. S2). In this structure, we also confirmed that the arsenic atom is bound in the same manner as described above for oxidized $D g$ AOR obtained with method 1 [24]. As the

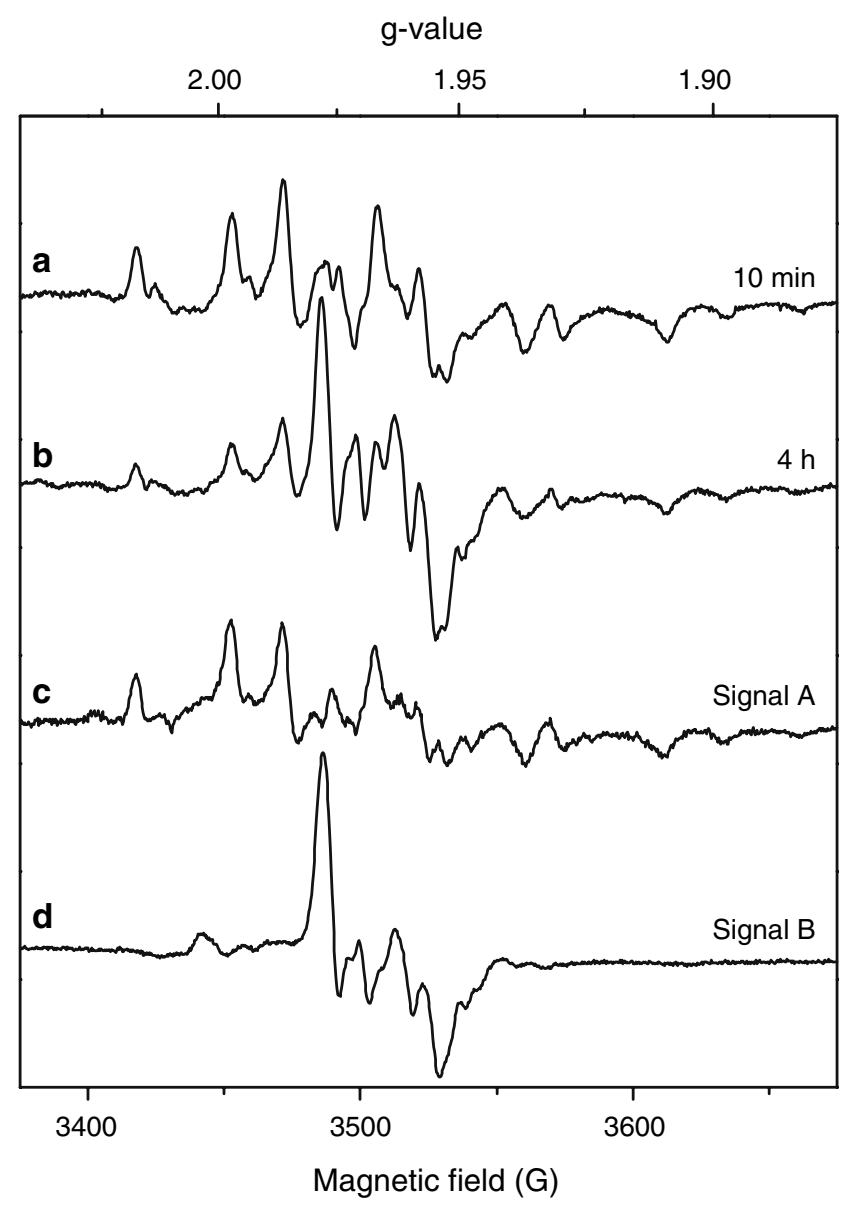

Fig. 4 EPR spectra of polycrystalline $D g$ AOR inhibited with $\mathrm{AsO}_{2}^{-} \cdot a$ Reduced with sodium dithionite for $10 \mathrm{~min}$ showing signal A. $b$ Same as $a$ but after 4-h reduction showing overlapped signals A and B. $c$ Signal A [24] and $d$ signal B from DgAOR in frozen solutions are included for comparison. The contribution of the resting signal (see text) has been subtracted in spectra $a$ and $c$
EPR experiments conducted on the sample giving signal A do not show solvent-exchangeable protons coupled to molybdenum, we concluded that the coordination around molybdenum of this complex must be similar to that shown in Fig. 1g.

Longer reduction $(4 \mathrm{~h})$ of polycrystalline AOR yields a mixture of signals $\mathrm{A}$ and $\mathrm{B}$ (Fig. 4, spectrum $\mathrm{b}$ ), as was also observed in experiments with AOR in solution. Integration of the signals indicates that about $60 \%$ of the $\mathrm{Mo}(\mathrm{V})$ species give signal $\mathrm{A}$, whereas $40 \%$ give signal $\mathrm{B}$ at the end of the incubation. Again, a single crystal was taken from the resulting EPR sample and transferred for an X-ray diffraction experiment. Although the resolution is lower owing to substantial crystal deterioration, the structure solved in this case $\left(D g\right.$ AOR-A* $\left.\mathrm{B}^{*}\right)$ is very similar to that of $D g$ AOR-A*, including the binding mode and the geometry of the arsenite moiety.

We also treated polycrystalline samples of $D g A O R$ by following the procedure used to obtain signal B. This was done by incubating arsenite-free crystals of $D g A O R$ in cryobuffer with an added excess of sodium dithionite in an EPR tube for $2 \mathrm{~h}$ under an argon atmosphere, followed by addition of sodium arsenite and further incubation (a reduction time of $2 \mathrm{~h}$ was used in this case rather than the $1 \mathrm{~h}$ used for the preparation of species B in solution to ensure the formation of species B). Although we could reproduce signal B by EPR from this sample, the conditions used caused the crystals to deteriorate and rendered them unsuitable for the collection of a full dataset.

\section{EPR saturation studies}

The low-temperature spectra $(20 \mathrm{~K})$ obtained in samples with signals $\mathrm{A}$ and $\mathrm{B}$ show in addition to the $\mathrm{Mo}(\mathrm{V})$ signal the EPR signal associated with the two [2Fe-2S] clusters (FeS I and FeS II) (Fig. 5 for DdAOR and Fig. S3 for $D g$ AOR). Both EPR signals A and B show temperature-dependent splittings produced by magnetic coupling with FeS I, as observed in dithionitereduced samples of the enzymes of the XO family [25]. Magnetic interactions depending on temperature occur when one of the species (FeS I) of the interacting pair has a relaxation rate $\left(T_{1}\right)$ faster than the other one $[\mathrm{Mo}(\mathrm{V})]$, which produces an enhancement of the relaxation rate of the slowly relaxing paramagnetic center and, consequently, a temperature dependence of the splitting of the resonance lines [25, 26, 43, 44]. The parameters and the temperature dependence of the EPR signals associated with the FeS centers in samples with signal B are similar to those obtained in reduced samples of as-purified $D g A O R$, indicating no structural 


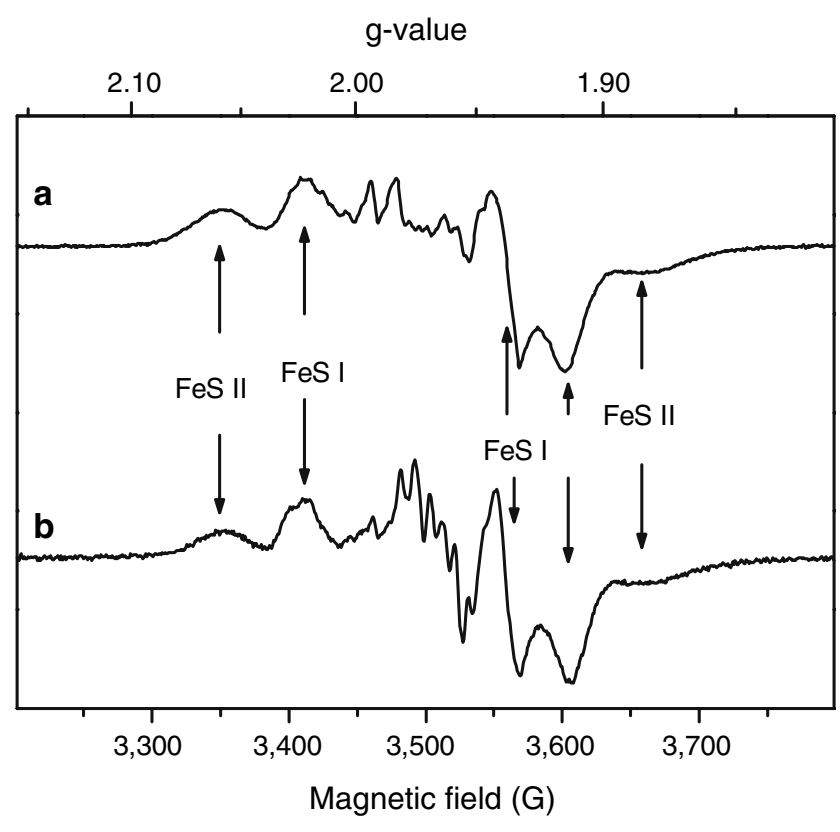

Fig. 5 EPR signals of arsenite-inhibited AOR at 20 K. $a$ Signal $\mathrm{A}$ and $b$ signal B from DdAOR. Note that both $\mathrm{Mo}(\mathrm{V})$ ion signals are further split by magnetic interaction with FeS I. The positions of the principal $g$ values of the FeS centers are indicated

changes in the FeS clusters (not shown). The Mo(V) signal obtained in samples with signal $\mathrm{A}$ are spread over a wider magnetic field region overlapping with part of the FeS I signal, which makes it difficult to clearly evaluate the EPR parameters. However, the positions of the signals suggest that both FeS I and FeS II centers show no significant changes in the two arsenite-inhibited forms of $D g$ AOR and $D d$ AOR.

Air exposure of the dithionite-reduced samples of both samples (signals A and B) oxidizes completely the $\mathrm{FeS}$ clusters to a diamagnetic state but leaves the $\mathrm{Mo}(\mathrm{V})$ species reduced. This can be advantageously used to evaluate changes in the relaxation properties of the $\mathrm{Mo}(\mathrm{V})$ species. Figure 6 shows the saturation behavior of the $\mathrm{Mo}(\mathrm{V})$ signal at $140 \mathrm{~K}$ in reduced and air-reoxidized samples. The $\mathrm{Mo}(\mathrm{V})$ species are less saturated at high powers in the presence of reduced FeS I than in the oxidized samples, which indicates that FeS I enhances its relaxation properties. The similar saturation behavior for both signals A and B suggests that the magnetic interaction also is similar in both cases.

\section{Discussion}

Our work shows that the inhibition of AOR with arsenite yields two different paramagnetic species

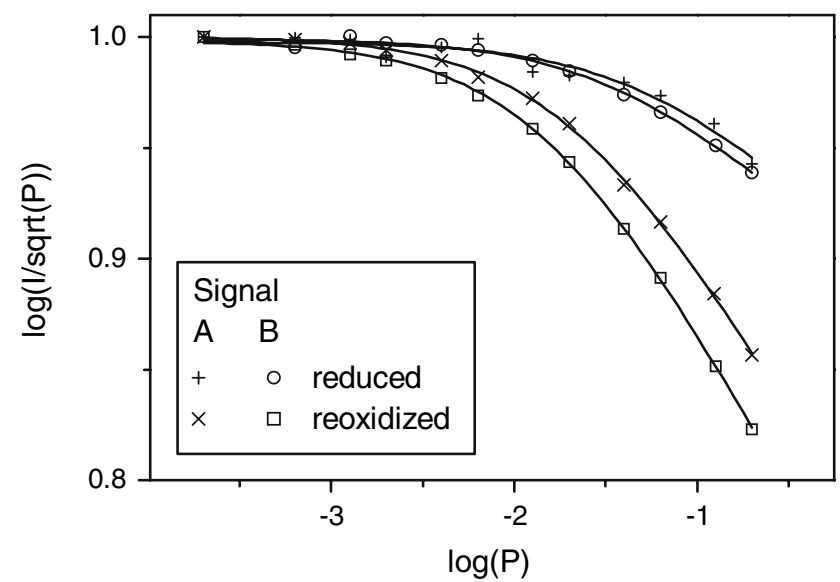

Fig. 6 Normalized power dependence plot of reduced and airreoxidized arsenite-inhibited EPR signals A (from [24]) and B at $140 \mathrm{~K}$ in $D g A O R$. The equation $y=P_{1}-\left(P_{2} / 2\right) \log \left(1+P / P_{3}\right)$ was fitted to the data. The intensity of the $3,450-\mathrm{G}$ peak for signal $A$ and the 3,485-G peak for signal B (depicted in Fig. 2) was used for the data, but all the strong peaks show the same saturation behavior. Microwave power $(P)$ in watts

depending on the initial oxidation state of the molybdenum atom. EPR signal A is obtained from arsenitereacted oxidized enzyme in solution, in which molybdenum is present as $\mathrm{Mo}(\mathrm{VI})$ ions, and which is subjected to dithionite reduction. In contrast, signal $\mathrm{B}$ is obtained from dithionite-reduced samples, which have molybdenum mostly as Mo(IV) ions, and which are treated with arsenite. X-ray data taken at different resolution levels of crystals grown from solution containing the EPR-active species (DgAOR-B, $D d$ AORA and DdAOR-B) confirm that in the two inhibited forms the arsenic atom interacts with molybdenum through an oxygen atom $\left(\mathrm{O}_{\mu}\right.$ in Table 3$)$ at the catalytic labile site.

Since both inhibited forms subjected to the same reducing conditions have different EPR properties, they should correspond to two different Mo-As complexes, in which the main structural difference is an equatorial oxygen ligand $\left(\mathrm{O}_{\mathrm{eq}}\right.$ in Table 3$)$ present as a hydroxyl/water ligand in species B but as an oxo ligand in species A (Fig. 7). Although the resolution of the crystallographic data is not sufficient for a definite structural confirmation of this point, EPR data indicate the presence of solvent-exchangeable protons in species B but not in species A, which must be associated with the $\mathrm{O}_{\text {eq }}$ ligand.

That species $\mathrm{A}$ and $\mathrm{B}$ are different complexes is also evidenced from the experiment in which samples showing signal A produce signal B after longer dithionite reductions without changes in the intensity of signal A. The X-ray data show that the As atom was found at $35 \%$ occupancy, similar to what was reported for the 

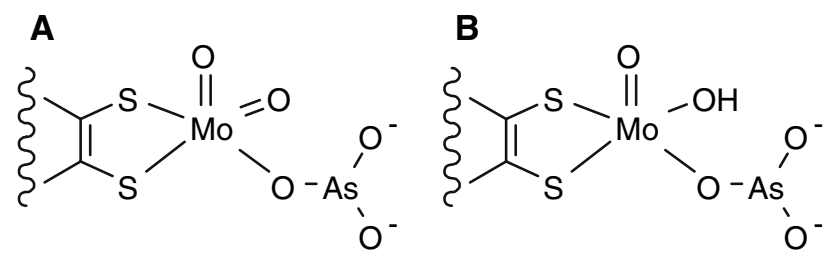

Fig. 7 Suggested geometries for species A and B. A corresponds to the previously published structure of arsenite-inhibited AOR [24], while $B$ corresponds to the structure presented in this work. The dithiolene ligand on the molybdenum atom is the pyranopterin depicted in Fig. $1 \mathrm{~h}$

previous structure of $D g$ AOR obtained with method 1 [24]. However, the two 2-propanol molecules, which were observed in the desulfo structure obtained from as-purified $D g$ AOR $[7,8]$, are also present in the arsenite-inhibited structure of $D g$ AOR-B, in contrast to the arsenite-inhibited $D g$ AOR obtained with method 1. This occurs because the protein sample was exposed to arsenite before the crystallization experiment and, therefore, the harvesting buffer did not need to be replaced with a 2-propanol-depleted solution. The 2propanol molecule close to the active site in the $D g$ AOR-B structure was found at $65 \%$ occupancy, as its binding site overlaps with that occupied by the arsenite moiety. A striking observation is that the Mo was present at full occupancy in structures from crystals grown using method 3 , whereas it was present at half occupancy in the structure of $D g$ AOR obtained with method 1. According to the Mo and As occupation levels of the crystals obtained with method 1, approximately $70 \%$ of the Mo sites have As atoms bound to them, which give signal A on reduction. In this context, the arsenic-free $D g A O R$ molecules, after reduction, would react with arsenite to give signal $\mathrm{B}$ (note that the single crystals are conserved in a soaking solution containing arsenite). This Mo redox state dependent behavior of AORs toward arsenite was also observed in corresponding EXAFS studies of arsenite-inhibited XO [28]. These studies showed that the arsenic interacts with the enzyme in its dithionite-reduced form but failed to show interaction with the oxidized form. The latter observation is in contradiction with our results which clearly show that arsenite reacts with both oxidized and dithionite-reduced forms of the enzyme.

X-ray data of the crystal DgAOR-A* (see supplementary material), complemented with EPR studies (Fig. 4), demonstrate that the sulfido ligand is not involved in the Mo-As complex giving DgAOR signal A and that it, therefore, is not required to give this signal. This result is in contradiction with earlier EPR [22] and EXAFS [28] data of dithionite-reduced arseniteinhibited XO that suggested the presence of the sulfido ligand in a bridging position between Mo and As atoms (Fig. 1). Despite the similar structural and EPR properties of XO and AORs from sulfate-reducing bacteria, it seems that both enzymes have different behavior towards arsenite, which raises many new questions regarding the active site configuration of AORs (e.g., the presence of the sulfido ligand) that need to be resolved in order to fully understand the chemistry behind these proteins.

Apart from the arsenite moiety coordinated to molybdenum, all the structures of arsenite-inhibited forms of AORs show no significant differences from the structures of the desulfo forms of the enzyme [7, 8]. This suggests that the proposed electron transfer pathway connecting the Mo atom and the two FeS centers is not modified upon inhibition. This was confirmed from EPR spectra of the FeS centers (Fig. 5) and EPR saturation studies (Fig. 6), which showed that the magnetic coupling between paramagnetic centers is present in both arsenite-inhibited forms.

Two recent crystal structures of QOR and XO with a bound substrate analog both showed a sulfur ligand in an equatorial position $[13,14]$. This ligand is believed to abstract the hydrogen from the carbon atom to be hydroxylated on the substrate, and its placement in an equatorial position should favor that process. Our data in AORs show that the arsenite moiety is coordinated in a similar manner to that of the substrate analog in the XO structure: (1) the Mo-O-As angle is similar to that evaluated for the Mo-O-C angle in $\mathrm{XO}$ $\left(130^{\circ}\right.$ vs. $\left.152^{\circ}\right)$ [13] and (2) one of the oxygen atoms bound to the arsenic atom is within hydrogen-bonding distance of the carboxylate group of Glu869, which is believed to be important in positioning the substrate molecule during catalysis. We also showed that the equatorial oxo ligand of the as-prepared enzyme, which should be a sulfido ligand in the active enzyme, is susceptible to protonation when the molybdenum is present mostly as Mo(IV) ions and the substrate binding site is blocked by the inhibitor. This result is also in line with the structure of the reduced form of substrate-bound $\mathrm{XO}$, in which the equatorial sulfido ligand is detected as a - $\mathrm{SH}$ group [13]. The evidence of proton chemistry at this position for an enzyme of the XO family gives additional support to the aforementioned abstraction mechanism.

EPR experiments on oriented single crystals complemented with X-ray data are usually used in solidstate chemistry and, because of some experimental limitations such as small crystal size and excessive number of non-equivalent magnetic sites, less frequently in proteins. This methodology can be used for different purposes. One of them is to determine the 
orientation of the $g$ tensor with respect to the molecular frame and/or to evaluate weak magnetic interactions between centers [45]. Another possibility is that reported by Högbom et al. [46], in which the method was used to evaluate changes in the orientation of a paramagnetic center. The method that we describe in this paper differs with respect to that mentioned before in that we used a non-oriented polycrystalline sample to assign the different EPR signals, which were initially detected in solution, to the molecular structure of the paramagnetic species. In principle, this is applicable to any system that contains an EPR-active center and that is suitable for single-crystal X-ray diffraction analysis. However, the success of the method depends on the possibility for diffusion of reactants into the crystals and whether the crystals remain suitable for diffraction analysis after repeated thawing and freezing and after exposure to the reactant(s) (e.g., dithionite), which in this case was introduced to develop the desired EPR signal. The latter could be a limitation in the case of protein crystallography, owing to reduction of the diffraction limit caused by crystal lattice decay. If, however, this loss of resolution is not severe, geometrical information on these centers can usually still be obtained since most paramagnetic centers are relatively heavy compared with the surrounding (protein) atoms. Note that the method can be also used with systems that already have an EPR signal, and addition of reagents in these cases can be used to monitor changes in the paramagnetic species. The success of the method will depend on the properties of the system under study and can probably only be assessed on the basis of trial and error. Although a more efficient way to grow and collect a substantial number of crystals than that described here would improve the ease of applying the technique, little effort or material is needed to apply the method. The experiments described here are therefore readily performed and we strongly feel that the additional information they provide justifies the effort.

Acknowledgements A.T. and D.R.B. thank the Fundação para a Ciência e Tecnologia, Portugal, for funding (grants SFRP/ BPD/5689/2001 and SFRH/BPD/20358/2004/E031, respectively, supported by the European Social Funding within the III Communitarian Support Board). D.R.B. and M.J.R. thank the EU (project number HRRN-CT-1999-00084) and the beamline scientists of the ID14-4 and BM14 beamlines of the ESRF in Grenoble for their assistance. C.D.B. and J.J.G.M. thank SECYT (Argentina) and GRICES (Portugal) for a bilateral collaborative grant. This work was supported by projects EC HPRN-CT-199900084, POCTI/1999/BME/35078, POCTI/1999/BME/36152 and POCTI/QUI/57641/2004 in Portugal, and by SEPCyT:PICT 2003-06-13872, CONICET PIP 5370/2005 and CAI + D-UNL in Argentina. C.D.B is a member of CONICET (Argentina).

\section{References}

1. Hille R (1996) Chem Rev 96:2757-2816

2. Romão MJ, Knäblein J, Huber R, Moura JJG (1997) Prog Biophys Mol Biol 68:121-144

3. Garattini E, Mendel R, Romão MJ, Wright R, Terao M (2003) Biochem J 372:15-32

4. Brondino CD, Romão MJ, Moura I, Moura JJG (2006) Curr Opin Chem Biol 10:109-114

5. Brondino CD, Rivas MG, Romão MJ, Moura JJG, Moura I (2006) Acc Chem Res 39:788-796

6. Romão MJ, Cunha CA, Brondino CD, Moura JJG (2002) Met Ions Biol Syst 39:539-570

7. Romão MJ, Archer M, Moura I, Moura JJG, LeGall J, Engh R, Schneider M, Hof P, Huber R (1995) Science 270:11701176

8. Rebelo JM, Dias JM, Huber R, Moura JJG, Romão MJ (2001) J Biol Inorg Chem 6:791-800

9. Rebelo J, Macieira S, Dias JM, Huber R, Ascenso CS, Rusnak F, Moura JJG, Moura I, Romão MJ (2000) J Mol Biol 297:135-146

10. Enroth C, Eger BT, Okamoto K, Nishino T, Pai EF (2000) Proc Natl Acad Sci USA 97:10723-10728

11. Truglio JJ, Theis K, LeimKühler S, Rappa R, Rajagopalan KV, Kisker C (2002) Structure 10:115-125

12. Dobbek H, Gremer L, Kiefersauer R, Huber R, Meyer O (2002) Proc Natl Acad Sci USA 99:15971-15976

13. Okamoto $\mathrm{K}$, Matsumoto $\mathrm{K}$, Hille R, Eger BT, Pai EF, Nishino T (2004) Proc Natl Acad Sci USA 101:7931-7936

14. Bonin I, Martins BM, Purvanov V, Fetzner S, Huber R, Dobbek H (2004) Structure 12:1425-1435

15. Turner N, Barata BAS, Bray RC, Deistung J, LeGall J, Moura JJG (1987) Biochem J 243:755-761

16. Duarte RO, Archer M, Dias JM, Bursakov S, Huber R, Moura I, Romão MJ, Moura JJG (2000) Biochem Biophys Res Commun 268:745-749

17. Barata BAS, LeGall J, Moura JJG (1993) Biochemistry 32:11559-11568

18. Duarte RO (2002) PhD thesis, Universidade Nova de Lisboa, Lisbon

19. Huber R, Hof P, Duarte RO, Moura JJG, Moura I, Liu M-Y, LeGall J, Hille R, Archer M, Romão MJ (1996) Proc Natl Acad Sci USA 93:8846-8851

20. Massey V, Edmondson D (1970) J Biol Chem 245:6595-6598

21. Hille R (1997) J Biol Inorg Chem 2:804-809

22. George GN, Bray RC (1983) Biochemistry 22:1013-1021

23. Hille R, Stewart RC, Fee JA, Massey V (1983) J Biol Chem 258:4849-4856

24. Boer DR, Thapper A, Brondino CD, Romão MJ, Moura JJG (2004) J Am Chem Soc 126:8614-8615

25. Caldeira J, Belle V, Asso M, Guigliarelli B, Moura I, Moura JJG, Bertrand P (2000) Biochemistry 39:2700-2707

26. Andrade SLA, Brondino CD, Feio MJ, Moura I, Moura JJG (2000) Eur J Biochem 267:2054-2061

27. Thapper A, Rivas MG, Brondino CD, Ollivier B, Fauque G, Moura I, Moura JJG (2006) J Inorg Biochem 100:44-50

28. Cramer SP, Hille R (1985) J Am Chem Soc 107:8164-8169

29. Moura JJG, Xavier AV, Bruschi M, LeGall J, Hall DO, Cammack R (1976) Biochem Biophys Res Commun 72:782789

30. Moura JJG, Xavier AV, Cammack R, Hall DO, Bruschi M, LeGall J (1978) Biochem J 173:419-425

31. Nilges MJ (1979) PhD thesis, University of Illinois, Urbana

32. Maurice AM (1980) PhD thesis, University of Illinois, Urbana 
33. Romão MJ, Barata BA, Archer M, Lobeck K, Moura I, Carrondo MA, LeGall J, Lottspeich F, Huber R, Moura JJG (1993) Eur J Biochem 215:729-732

34. Leslie AGW (1992) Joint CCP4+ESF-EAMCB newsletter on protein crystallography 26

35. Collaborative Computational Project Number 4 (1994) Acta Crystallogr Sect D 50:760-763

36. Murshudov GN, Vagin AA, Dodson EJ (1997) Acta Crystallogr Sect D 53:240-255

37. Kabsch W (1993) J Appl Crystallogr 26:795-800

38. Pignol D, Gaboriaud C, Fontecilla-Camps JC, Lamzin VS, Wilson KS (1996) Acta Crystallogr Sect D 52:345-355

39. Greenwood RJ, Wilson GL, Pilbrow JR, Wedd AG (1993) J Am Chem Soc 115:5385-5392

40. Moura JJG, Barata BAS (1994) Methods Enzymol 243:24-42
41. Bray RC, Knowles PF, Pick FM, Vänngård T (1968) Biochem J 107:601-602

42. Gutteridge S, Tanner SJ, Bray RC (1978) Biochem J 175:887-897

43. Hirsh DJ, Beck WF, Innes JB, Brudvig GW (1992) Biochemistry 31:532-541

44. Hirsh DJ, Beck WF, Lynch JB, Que L Jr, Brudvig GW (1992) J Am Chem Soc 114:7475-7481

45. Schveigkardt JM, Rizzi AC, Piro OE, Castellano EE, Costa de Santana R, Calvo R, Brondino CD (2002) Eur J Inorg Chem 2002:2913-2919

46. Högbom M, Galander M, Andersson M, Kolberg M, Hofbauer W, Lassmann G, Nordlund P, Lendzian F (2003) Proc Natl Acad Sci USA 100:3209-3214 